Commun. math. Phys. 7, 225-233 (1968)

\title{
Boundary Values of Analytic Functions
}

\author{
Florin Constantinescu
}

Department of Theoretical Physics

University of Cluj - Romania

Received July 15, 1967

Abstract. It is known that a complex - valued continuous function $S(x)$ as well as a Schwartz distribution on the real axis can be extended in the complex plane minus the support of $S$ to an analytic function $\hat{S}(z)$. In the case of a continuous function the jump of $\hat{S}(z)$ on the real axis represents exactly $S(x)$ :

$$
\lim _{\varepsilon \rightarrow 0+}[\hat{S}(x+i \varepsilon)-\hat{S}(x-i \varepsilon)]=S(x) .
$$

We call regular a point $x$ on the support of $S$ such that $\lim _{\varepsilon \rightarrow 0+}[\hat{S}(x+i \varepsilon)-$ $-\hat{S}(x-i \varepsilon)]$ exists. Conditions are found for the existence of regular points on the support of a distribution. It is possible to call this limit (if this exists) the value $S(x)$ of the distribution $S$ in the point $x$. Properties of this type occur in the theory of dispersion relations.

\section{\$1. Introduction}

Let $S(x)$ be a complex-valued continuous function with compact support on the real axis $R$. The function $S(x)$ can be extended in the complex plane $[1-3]$ under the form of a local analytic function $\hat{S}(z)$ throughout the entire complex plane minus the support of $S$, such that the jump on the real axis is exactly $S(x)$ :

$$
\lim _{\varepsilon \rightarrow 0+}[\hat{S}(x+i \varepsilon)-\hat{S}(x-i \varepsilon)]=S(x) \text {. }
$$

Relations of the form (1) frequently occur in the study of analytical properties of the scattering amplitude as well as in the theory of dispersion relations $[4-5]$.

The idea to extend functions on the real axis to the complex plane, in such a way that the relation (1) be verified, is an older one. Excellent expositions of this problem can be found in $[6-8]$. In the case when the function $S(x)$ has a compact support, the analytic continuation $\hat{S}(z)$ can be chosen under integral form

$$
\hat{S}(z)=\frac{1}{2 \pi i} \int_{-\infty}^{+\infty} \frac{S(t)}{t-z} d t
$$


called the Cauchy representation of $S$. Representations of type (2) as well as relations of type (1) can also occur in other hypothesis than those given here. Particularly the limit (1) exists if $S(x)$ is piecewise continuous ${ }^{1}$ and $x$ is a point of continuity on the compact support of $S$. But if $x$ is not a continuity point of $S$, the relation (1) takes the following form

$$
\lim _{\varepsilon \rightarrow 0+}[\hat{S}(x+i \varepsilon)-\hat{S}(x-i \varepsilon)]=\frac{1}{2}\left[S\left(x_{+}\right)+S\left(x_{-}\right)\right] .
$$

In this paper we are concerned with finding some more general hypothesis under which $S(x)$ has stronger discontinuities when however a relation of type (3) can be given. It is more convenient to treat this problem within Schwartz distributions.

It is known [2] that $S \in\left(\mathscr{D}^{\prime}\right)$ being a given distribution, it can be extended in the whole complex plane minus the support of $S$, so that

$$
\lim _{\varepsilon \rightarrow 0+} \int_{-\infty}^{\infty}[\hat{S}(x+i \varepsilon)-\hat{S}(x-i \varepsilon)] \varphi(x) d x=\langle S, \varphi\rangle
$$

for all $\varphi \in(\mathscr{D})$.

The idea to continue distributions to complex variable functions appears in [9] in connection with the continuation of Fourier transforms of distributions. Similarly the Wightman functions [10] ( $n$-fold vacuum expectation values) represent a particular case of continuation of distribution through the complex variable functions. Similar techniques are used in mathematical theory of dispersion relations [11-13]. Excellent treatises of this problem can be found in [14].

Although the relation (4) is valid we cannot state generally anything relatively to the existence of the limit

$$
\lim _{\varepsilon \rightarrow 0+}[\hat{S}(x+i \varepsilon)-\hat{S}(x-i \varepsilon)] .
$$

Nevertheless it might be possible that independently of (4), to exist the limit (5) for certain points on the support of $S$. Especially this is true if the distribution $S$ is reduced to a piecewise function with compact support. More general cases of the existence of the limit (5) will be pointed out in $\S 3$ (theorems 3 and 4 ). In $\S 4$ will be shown that the results obtained are in close relation with the problem of the value in a point of a distribution [15-16] and at the same time will be pointed out the possibilities of generalization of these results. In $\S 2$ are given general theorems for representation of distributions by analytic functions in the complex plane [2] and a corollary which shows that the results obtained in $\S 3$ are of a local nature.

1 The function $f(t)$ is called piecewise continuous if and only if $f$ is continuous except for a finite number of points at which the right-hand limite $f\left(x_{+}\right)$ $=\lim _{\delta \rightarrow 0+} f(x+\delta)$ and the left-hand limit $f\left(x_{-}\right)=\lim _{\delta \rightarrow 0-} f(x+\delta)$ exist. 


\section{\$ 2. Representation of Distributions by Analytic Functions}

Theorem 1. Let $S$ be a distribution with compact support. Then the Cauchy representation $\hat{S}(z) \equiv \frac{1}{2 \pi i}\left\langle S, \frac{1}{t-z}\right\rangle$ exists and it is an analytic function of $z$ in the complement of the support of $S$. Also, the derivatives of $\widehat{S}(z)$ and $S$ are related by the equality

$$
\left(\frac{d^{n}}{d z^{n}} \hat{S}(z)\right)=\hat{S}^{(n)}(z)=\frac{n !}{2 \pi i}\left\langle S, \frac{1}{(t-z)^{n+1}}\right\rangle .
$$

Theorem 2. Let $S \in\left(\mathscr{D}^{\prime}\right)$. Then there exists a function $\hat{S}(z)$, analytic in the z-plane, except on the real axis, such that

$$
S=\lim _{\varepsilon \rightarrow 0+}[\hat{S}(x+i \varepsilon)-\hat{S}(x-i \varepsilon)],
$$

the limit being taken in the sense of distributions. Moreover, for any such $\hat{S}, \hat{S}$ is analytic in the complex plane minus the support of $S$.

Corollary. Let $S$ and $T$ be two distributions which are equal on an open set $O$ of the real axis and suppose we have the representations

$$
\begin{aligned}
& S=\lim _{\varepsilon \rightarrow 0+}[\hat{S}(x+i \varepsilon)-\hat{S}(x-i \varepsilon)] \\
& T=\lim _{\varepsilon \rightarrow 0+}[T(x+i \varepsilon)-\hat{T}(x-i \varepsilon)],
\end{aligned}
$$

the limit being taken in the sense of distributions. Then from the existence of the limit

$$
S(x)=\lim _{\varepsilon \rightarrow 0+}[\hat{S}(x+i \varepsilon)-\hat{S}(x-i \varepsilon)]
$$

in a point $x \in O$, it follows the existence of the limit

in the point $x$.

$$
T(x)=\lim _{\varepsilon \rightarrow 0+}[\hat{T}(x+i \varepsilon)-\hat{T}(x-i \varepsilon)]
$$

This corollary follows immediately observing that the difference $S-T$ is zero on $O$ and consequently taking into account theorem 2 , the function $\hat{S}(z)-\hat{T}(z)$ is analytic over $O$.

\section{$\$$ 3. Regular Points of Distributions}

Returning to the problem which we pointed out in the introduction, we call the point $x$ a regular point of the distributions $S$ if the limit (5) exists. It is evident in the case in which the distribution $S$ is reduced to a continuous functon with compact support that each point is a regular one. There are some more general cases of existence of regular points as it is shown by the following theorem:

Theorem 3. The point $x$ is a regular point of the distribution $S \in\left(\mathscr{D}^{\prime}\right)$ if there exists an integer $n \geqq 0$ and a continuous function $F(t)$ so that $F^{(n)}(t)=S(t)$ the derivative being taken in the sense of distributions and if 
also exists

$$
\lim _{t \rightarrow x} n ! \frac{F(t)}{(t-x)^{n}}=C
$$

Limit (5) is then equal with $C$.

Proof. It is enough to prove our statement in the case of a distribution with real values because we can split the distribution with complex values in a real part and in an imaginary one. On the other hand taking into account the corollary from $\S 2$, we can consider that the distribution $S$ and the function $F$ are with compact support.

Hence by theorem 1 we have

$$
\begin{aligned}
\left\langle S, \frac{1}{t-z}\right\rangle & =\left\langle F^{(n)}, \frac{1}{t-z}\right\rangle=\frac{n !}{2 \pi i}\left\langle F, \frac{1}{(t-z)^{n+1}}\right\rangle= \\
& =\frac{n !}{2 \pi i} \int_{-\infty}^{\infty} \frac{F(t)}{(t-z)^{n+1}} d t .
\end{aligned}
$$

Consequently

$$
\begin{aligned}
& \hat{S}(x+i \varepsilon)-\hat{S}(x-i \varepsilon)=\frac{n !}{2 \pi i} \int_{-\infty}^{\infty} F(t) \times \\
& \times\left[\frac{1}{(t-x-i \varepsilon)^{n+1}}-\frac{1}{(t-x+i \varepsilon)^{n+1}}\right] d t .
\end{aligned}
$$

Now being given a number $\varepsilon^{\prime}>0$, in accordance with the hypothesis, we can have $\delta>0$, so that

$$
\left|n ! \frac{F(t)}{(t-x)^{n}}-C\right|<\varepsilon^{\prime} \text { for }|t-x|<\delta .
$$

Let $M$ be a bound for $|F(t)|$. We can write

$$
\begin{aligned}
\mid \hat{S}(x+ & i \varepsilon)-\hat{S}(x-i \varepsilon)-C|\leqq| \frac{n !}{2 \pi i} \int_{x-\delta}^{x+\delta} \frac{F(t)}{(t-x)^{n}}(t-x)^{n} \times \\
& \times\left[\frac{1}{(t-x-i \varepsilon)^{n+1}}-\frac{1}{(t-x+i \varepsilon)^{n+1}}\right] d t-C \mid+ \\
+ & \frac{n ! M}{2 \pi} \int_{|t-x| \geqq \delta}\left|\frac{1}{(t-x-i \varepsilon)^{n+1}}-\frac{1}{(t-x+i \varepsilon)^{n+1}}\right| d t .
\end{aligned}
$$

Stopping at the first term of the right side of the inequality (12) we have

$$
\begin{aligned}
& \left|\frac{n !}{2 \pi i} \int_{x-\delta}^{x+\delta} \frac{F(t)}{(t-x)^{n}}(t-x)^{n}\left[\frac{1}{(t-x-i \varepsilon)^{n+1}}-\frac{1}{(t-x+i \varepsilon)^{n+1}}\right] d t-C\right| \leqq \\
& \quad \leqq \frac{\varepsilon}{2 \pi} \int_{x-\delta}^{x+\delta}\left|(t-x)^{n}\left[\frac{1}{(t-x-i \varepsilon)^{n+1}}-\frac{1}{(t-x+i \varepsilon)^{n+1}}\right]\right| d t+\quad(13) \\
& \quad+|C| \cdot\left|\frac{1}{2 \pi} \int_{x-\delta}^{x+\delta}(t-x)^{n}\left[\frac{1}{(t-x-i \varepsilon)^{n+1}}-\frac{1}{(t-x+i \varepsilon)^{n+1}}\right] d t-1\right| .
\end{aligned}
$$


We may remark that by a change of variable the integrals of the second term get the forms

We have

$$
\int \frac{t^{n}}{(t+i y)^{n+1}} d t, \quad y= \pm \varepsilon
$$

$$
\begin{aligned}
\int \frac{t^{n}}{(t+i y)^{n+1}} d t & =\int \frac{(t+i y-i y)^{n}}{(t+i y)^{n+1}} d t=\ln (x+i y)- \\
& -\sum_{k=1}^{n} \frac{\left(\begin{array}{l}
n \\
k
\end{array}\right)}{k} \frac{(-i y)^{k}}{(t+i y)^{k}}
\end{aligned}
$$

and consequently

$$
\begin{gathered}
\int_{-\delta}^{\delta} t^{n}\left[\frac{1}{(t-i \varepsilon)^{n+1}}-\frac{1}{(t+i \varepsilon)^{n+1}}\right] d t=2 \int_{0}^{\delta} t^{n}\left[\frac{1}{(t-i \varepsilon)^{n+1}}-\frac{1}{(t+i \varepsilon)^{n+1}}\right] d t \\
=2\left[2 \operatorname{arctg} \frac{\delta}{\varepsilon}-\frac{\left(\begin{array}{l}
n \\
k
\end{array}\right)}{k} \frac{(i \varepsilon)^{k}}{(\delta-i \varepsilon)^{k}}+\frac{\left(\begin{array}{l}
n \\
k
\end{array}\right)}{k}-\frac{(-i \varepsilon)^{k}}{(\delta+i \varepsilon)^{k}}\right]
\end{gathered}
$$

where by arctg we denoted the principal value. From (15) we get

$$
\lim _{\varepsilon \rightarrow 0+} \int_{-\delta}^{\delta} t^{n}\left[\frac{1}{(t-i \varepsilon)^{n+1}}-\frac{1}{(t+i \varepsilon)^{n+1}}\right] d t=2 \pi .
$$

Hence the second term of the right side of the inequality (13) tends to zero when $\varepsilon \rightarrow 0$.

For the other term we observe that

$$
\begin{gathered}
\int_{x-\delta}^{x+\delta}\left|(t-x)^{n}\left[\frac{1}{(t-x-i \varepsilon)^{n+1}}-\frac{1}{(t-x+i \varepsilon)^{n+1}}\right]\right| d t \\
=\int_{-\delta}^{\delta}\left|t^{n}\left[\frac{1}{(t-i \varepsilon)^{n}}-\frac{1}{(t+i \varepsilon)^{n}}\right]\right| d t \\
=\int_{-\delta}^{\delta}\left|\frac{t^{n}}{i}\left[\frac{1}{(t-i \varepsilon)^{n+1}}-\frac{1}{(t-i \varepsilon)^{n+1}}\right]\right| d t=\int_{-\delta}^{\delta}|\omega(t)| d t
\end{gathered}
$$

where the function

$$
\omega(t)=\frac{t^{n}}{i}\left[\frac{1}{(t-i \varepsilon)^{n+1}}-\frac{1}{(t+i \varepsilon)^{n+1}}\right]
$$

is a real one. For $n=0,1$ the function $\omega(t)$ does not change the sign on the whole axis and consequently taking into account (16), we have

$$
\lim _{\varepsilon \rightarrow 0+} \int_{-\delta}^{\delta}|\omega(t)| d t=\lim _{\varepsilon \rightarrow 0+} \int_{-\delta}^{\delta} \omega(t) d t=2 \pi .
$$

Let us suppose now that $n>1$. The function $\omega(t)$ is symmetric and we have $\omega(0)=0$. The non-vanishing solutions of $\omega(t)=0$ are obtained for

$$
(t+i \varepsilon)^{n+1}=(t-i \varepsilon)^{n+1}
$$


hence, these are simple roots of the form $t_{r}=\gamma_{r} \varepsilon ; r=-m,-m+1, \ldots$, $-1,1, \ldots, m-1, m$ where $2 m$ represents their number. The numbers $\gamma_{r}$ depend only on $n$. Hence $\delta$ being chosen in this way, we can take $\varepsilon$ sufficiently small so that all the zeros of the function $\omega(t)$ be situated in the interval $(-\delta, \delta)$. Consequently we can write

$$
\begin{gathered}
\int_{-\delta}^{\delta}|\omega(t)| d t=2 \int_{0}^{\delta}|\omega(t)| d t=2 \mid \int_{0}^{\gamma_{1} \varepsilon} \omega(t) d t- \\
-\int_{\gamma_{1} \varepsilon}^{\gamma_{2} \varepsilon} \omega(t) d t+\cdots+(-1)^{m-1} \int_{\gamma_{m-1} \varepsilon}^{\gamma_{m} \varepsilon} \omega(t) d t+(-1)^{m} \int_{\gamma_{m} \varepsilon}^{\infty} \omega(t) d t \mid .
\end{gathered}
$$

On the other hand from (14) we have

$$
\begin{aligned}
& \int_{\gamma_{r-1} \varepsilon}^{\gamma_{r} \varepsilon} \omega(t) d t= 2 \operatorname{arctg} \gamma_{r}-2 \operatorname{arctg} \gamma_{r-1}-\sum_{k=1}^{n} \frac{\left(\begin{array}{l}
n \\
k
\end{array}\right)}{k} \frac{i^{k-1}}{\left(\gamma_{r}-i\right)^{k}}+ \\
&+\sum_{k=1}^{n} \frac{\left(\begin{array}{l}
n \\
k
\end{array}\right)}{k} \frac{i^{k-1}}{\left(\gamma_{r-1}-i\right)^{k}}+\sum_{k=1}^{n}(-1)^{k} \frac{\left(\begin{array}{l}
n \\
k
\end{array}\right)}{k} \frac{i^{k-1}}{\left(\gamma_{r}+i\right)^{k}}- \\
&-\sum_{k=1}^{n}(-1)^{k} \frac{\left(\begin{array}{l}
n \\
k
\end{array}\right)}{k} \frac{i^{k-1}}{\left(\gamma_{r-1}+i\right)^{k}} .
\end{aligned}
$$

We observe that this results depends only on $n$ for all $r=1,2 \ldots$, $m\left(\gamma_{0}=0\right)$.

$\left.\gamma_{0}=0\right)$.
In the same way the integral $\int_{\gamma_{m}}^{\infty} \omega(t) d t$ is a finite one and depends only on $n$. Hence taking into account (19) and (22) for a given $n$ we can choose $\varepsilon$ so that the first term in the right side of the inequality (13) be smaller than $A \varepsilon^{\prime}$, where $A$ is a constant which depends only on $n$.

For the last term of (12) we obtain

$$
\begin{aligned}
\int_{|t| \geq \delta} \mid \frac{1}{(t-i \varepsilon)^{n+1}} & -\frac{1}{(t+i \varepsilon)^{n+1}}|d t=| \int_{-\infty}^{-\delta}\left[\frac{1}{(t-i \varepsilon)^{n+1}}-\frac{1}{(t+i \varepsilon)^{n+1}}\right] d t \mid+ \\
& +\left|\int_{\delta}^{\infty}\left[\frac{1}{(t-i \varepsilon)^{n+1}}-\frac{1}{(t+i \varepsilon)^{n+1}}\right] d t\right| \\
& =2\left|\int_{\delta}^{\infty}\left[\frac{1}{(t-i \varepsilon)^{n+1}}-\frac{1}{(t+i \varepsilon)^{n+1}}\right] d t\right|
\end{aligned}
$$

since, for $t \leqq-\delta$ and $t \geqq \delta$ the function

$$
i\left[\frac{1}{(t-i \varepsilon)^{n+1}}-\frac{1}{(t+i \varepsilon)^{n+1}}\right]
$$

does not change its sign. On the other hand it is easy to show that we 
have

$$
\lim _{\varepsilon \rightarrow 0+} \int_{\delta}^{\infty}\left[\frac{1}{(t-i \varepsilon)^{n+1}}-\frac{1}{(t+i \varepsilon)^{n+1}}\right] d t=0 .
$$

Consequently $\varepsilon^{\prime}$ and $\delta$ being once chose we can find an $\varepsilon$ sufficiently small so that the second term of the right side of (13) together with the second term of the right side of (12) are smaller than $\varepsilon^{\prime}$. From (12) we obtain

$$
|\hat{S}(x+i \varepsilon)-\hat{S}(x-i \varepsilon)-C| \leqq A \varepsilon^{\prime}+\varepsilon^{\prime}=(A+1) \varepsilon^{\prime} .
$$

Since $\varepsilon^{\prime}$ is any positive number it follows that

$$
\lim _{\varepsilon \rightarrow 0+}[\hat{S}(x+i \varepsilon)-\hat{S}(x-i \varepsilon)]=C .
$$

This proves our theorem.

The theorem 3 can be extended as follows.

Theorem 4. The point $x$ is regular point of the distribution $S \in\left(\mathscr{D}^{\prime}\right)$ if there exists an integer $n \geqq 0$ and a continues function $F(t)$ so that $F^{(n)}(t)$ $=S(t)$, the derivative being taken in the sense of distributions and exising also

$$
\lim _{t \rightarrow x+} n ! \frac{F(t)}{(t-x)^{n}}=C_{+}, \quad \quad \lim _{t \rightarrow x-} n ! \frac{F(t)}{(t-x)^{n}}=C_{-} .
$$

The limit (5) is then equal with $\frac{C_{+}+C_{-}}{2}$.

Proof. We have

$$
\begin{gathered}
\hat{S}(x+i \varepsilon)-\hat{S}(x-i \varepsilon)-\frac{C_{+}+C_{-}}{2}=\frac{n !}{2 \pi i} \int_{0}^{x+\delta} \frac{F(t)}{(t-x)^{n}}(t-x)^{n} \times \\
\times\left[\frac{1}{(t-x-i \varepsilon)^{n+1}}-\frac{1}{(t-x+i \varepsilon)^{n+1}}\right] d t-\frac{C_{+}}{2} \mid+\quad\left(12^{\prime}\right) \\
+\left|\frac{n !}{2 \pi i} \int_{x-\delta}^{0} \frac{F(t)}{(t-x)^{n}}(t-x)^{n}\left[\frac{1}{(t-x-i \varepsilon)^{n+1}}-\frac{1}{(t-x+i \varepsilon)^{n+1}}\right] d t-\frac{C_{-}}{2}\right|+ \\
+\frac{n ! M}{2 \pi} \int_{|t-x| \geqq \delta}\left|\frac{1}{(t-x-i \varepsilon)^{n+1}}-\frac{1}{(t-x+i \varepsilon)^{n+1}}\right| d t
\end{gathered}
$$

Now we can repeat step by step the proof of theorem 2 .

In the case in which the distribution $S \in\left(\mathscr{D}^{\prime}\right)$ is reduced to a piecewise continuous function, by using the theorems 3 and 4 we can obtain certain results found in [2]. In these cases $C$ represents the value $S(x)$ for a continuity point and $\frac{C_{+}+C_{-}}{2}$ is the "value" of this function for a discontinuity point. 


\section{$\$$ 4. The Value of a Distribution in a Point}

The notion of the value of the distribution in a point has been introduced in [15]. One can say that distribution $S(t)$ has a value in the point $x$ if there exists the limit

$$
\lim _{\alpha \rightarrow 0} S(\alpha t+x)=C
$$

in the sense of distributions and this limit is the constant distribution $C$. It was pointed out that from the existence of the limit (26) it follows its constancy. A necessary and sufficient condition for the existence of the value of a distribution in a point is given by

Theorem 5. The distribution $S \in\left(\mathscr{D}^{\prime}\right)$ has a value in the point $x$ if and only if there exists an integer $n \geqq 0$ and a continuous function $F(t)$ such that $F^{(n)}(t)=S(t)$, the derivative being taken in the sense of the distributions, and

$$
\lim _{t \rightarrow x} n ! \frac{F(t)}{(t-x)^{n}}=C .
$$

The value of the distribution $S$ in the point $x$ is then $S(x)=C$.

Theorem 5 may be stated in the following equivalent form [16].

Theorem 5'. The distribution $S \in\left(\mathscr{D}^{\prime}\right)$ has a value in the point $x$ if and only if there exists an integer $n \geqq 0$ and a continuous function $\Phi(t)$ so that $\Phi^{(n)}(t)=S(t)$, the derivative being given in the sense of the distribution and

$$
\lim _{x_{i} \rightarrow x, i=1,2, \ldots n} n !\left[x, x_{1}, x_{2}, \ldots, x_{n} ; \Phi\right]=C,
$$

the points $x_{1}, x_{2}, \ldots, x_{n}$ tending independently to $x^{2}$.

By comparing the theorems 2 and 4 we obtain

Theorem 6. Let $S \in\left(\mathscr{D}^{\prime}\right)$. If the distribution $S(t)$ has a value in the point $x$ this is a regular point and we have

$$
\lim _{\varepsilon \rightarrow 0+}[\hat{S}(x+i \varepsilon)-\hat{S}(x-i \varepsilon)]=S(x) .
$$

Particularly if there exists the derivative of a continuous function $F(t)$ in the point $x$, then there exists also the value in $x$ of the distribution $F^{\prime}(t)$ and it coincides with the value of the common derivative. Hence the point $x$ is a regular point for $F^{\prime}(t)$. The function

$$
F(t)=3 t^{2} \sin \frac{1}{t}-t \cos \frac{1}{t}
$$

offers an example in which the common derivative does not exist in the point 0 , and although there exists the value of the distribution $F^{\prime}(t)$ and therefore this point is a regular one for $F^{\prime}(t)$. From theorem $5^{\prime}$ it follows that the problem of the value of a distribution in a point and hence the problem of existence of regular points is in close connexion

2 With this occasion we remark that this condition is an essential one. Hence we cannot take $x_{1}=x_{2}=\cdots=x_{n}$ and after that $x_{1} \rightarrow x$. 
with the older problem of existence of a $n$-order direct derivative for a continuous function [16].

Theorem 4 shows that generally we can have regular points without existence of the limit (28). Therefore it is more convenient to call "value" of the distribution the limit $(5)$ if the point $x$ is a regular one.

We remark at last that our theorems may be extended to the case of several independent variables and to the representation of distribution by harmonic functions with complex values [17].

Acknowledgement. The autor is indebted to Professor L. SchwarTz for an illuminating suggestion regarding this paper. Also I would like to thank Mr. H. WIESLER for interesting discussions regarding this subject.

\section{References}

1. Bremermann, H. I., and L. Durand: J. Math. Phys. 2, 240 (1960).

2. - Distributions, complex variables and Fourier transforms. Reading (Mass.): Addison-Wesley Publishing Co., Inc. 1965.

3. GütTinger, W.: Fortschr. Physik 14, 483 (1966).

4. Roman, P.: Advanced quantum theory. Reading (Mass.): Addison-Wesley Publishing Co., Inc. 1965.

5. Barton, G.: Introduction to dispersion techniques in filed theory. New York: Benjamin Inc. 1965.

6. Carleman, T.: L'integrale de Fourier et questions qui s'y rattachent. Uppsala: Alqvist \& Wiksells Boktryckeri - A. - B. 1944.

7. Muskhelishvili, N. I.: Singular integral equations. Moscow: Fizmatgiz 1962.

8. GaKHov, F. D.: Boundary problems. Moscow: Fizmatgiz 1963.

9. Schwartz, L.: Medd. Lunds. Univ. Mat. Sem. Suppl. M. Riezz 196 (1952).

10. Streater, R., and A.S. Wightmax: PCT, spin and statistics and all that. New York: Benjamin Inc., 1964.

11. Bogolubov, N. N., V. B. Medvenev, and M. K. Polivanov: Problems in the theory of dispersion relations. Moscow: Fizmatgiz 1958.

12. Bremermann, H. I., R. Oehme, and J. G. Taylor: Phys. Rev. 109, 2178 (1958).

13. TAYLoR, J. G.: Ann. Phys., 5, 391 (1958).

14. Кӧтне, G.: Topologische lineare Räume I. Berlin: Springer 1960.

15. EoJasiewicz, S.: Studia Math., 16, 1 (1957).

16. Constantinescu, F.: Studia Math., 24, 7 (1964).

17. Bremermann, H. I.: J. Anal. Math. 14, 5 (1965).

Dr. F. Constantinescu

Department of Theoretical Physics

University of Cluj

Cluj, Romania 